\title{
VALORES NORMAIS DAS VELOCIDADES DE CONDUÇÃO NERVOSA EM UM GRUPO DE 101 PESSOAS
}

\author{
DALVA LIVRAMENTO GRANDINI* - J.A. MACIEL NÓBREGA** \\ YARA JULIANO ***
}

\begin{abstract}
RESUMO - Foram estudadas as medidas de condução nervosa em 101 pessoas normais, 53 do sexo masculino e 48 do feminino. A idade variou de 16 a 81 anos (média, 44 anos). Foram estudadas tanto a velocidade de condução sensitiva quanto a velocidade de condução motora nos nervos mais frequentemente avaliados em neurofisiologia clínica. Em relação ao fator idade, observou-se declínio significativo nas faixas etárias mais avançadas.
\end{abstract}

PALAVRAS CHAVE: velocidade de condução nervosa, valores normais, idade.

Normal nerve conduction velocities in a group of 101 persons.

SUMMARY - Motor and sensory nerve conduction velocities were analysed in a group of 101 normal Brazilians. The normal values were obtained considering the group age, the distal and proximal segments of each nerve, the differences in the right and left side for each nerve. A significant decline in the conduction was found for the elderly aged group.

KEY WORDS: nerve conduction velocity, normal parameters, ageing.

O primeiro estudo da velocidade de condução motora no homem foi realizado por Helmholtz em 1850, segundo Kimura 16. Em 1949, Dawson e Scott 7 abriram caminho para a aplicação diagnóstica das medidas das velocidades de condução sensitiva, estudando os nervos mediano e ulnar ao nível do punho. Estudos subsequentes foram realizados por autores como Simpson 21 e Gilliat e Thomas 10. Gilliat e Thomas 11 estudaram os nervos peroneal e poplíteo lateral, estimulando-os no tornozelo e registrando o potencial na cabeça da fíbula, em 39 pessoas, encontrando redução da velocidade de condução na faixa etária de 20 a 80 anos. La Fratta e Canestrari 17, estudando 128 pessoas na faixa etária de 23 a 91 anos, também relatam declínio da velocidade de condução sensitiva no nervo mediano com o passar da idade. Diversos autores realizaram estudos tanto para a avaliação da velocidade de condução sensitiva quanto para a motora realizando, ora alterações nas técnicas ora nos parâmetros de comparação. Behse e Buchthal 1 estabeleceram valores normais para a velocidade de condução sensitiva dos segmentos distais e proximais dos nervos tibiais posteriores, surais e peroneais em 71 pessoas com idades variando entre 15 e 72 anos. Checkles e col.3, Nelson 20 e Harding e Halar 15 realizaram estudos mais complexos, principalmente na avaliação do nervo ulnar, verificando em angulações do cotovelo as respectivas alterações nas velocidades de condução motora. Diversas pesquisas foram realizadas para se estudar a ação da temperatura sobre a velocidade de condução nervosa 5,12_14, referindo diminuição da velocidade de condução nervosa com a diminuição da temperatura tanto ambiental quanto das extremidades examinadas. Thies e col.23; Sing e Maini 22 e Longe 18 estudaram grupos raciais diferentes e não evidenciaram alterações significativas das velocidades de condução.

O objetivo deste estudo foi o de avaliar as medidas das velocidades de condução sensitiva e motora em um grupo de indivíduos brasileiros.

Trabalho do Setor de Neurofisiologia Clínica da Disciplina de Neurologia da Escola Paulista de Medicina (EPM): * Mestre em Neurologia; ** Professor Adjunto Doutor, Responsável pelo Setor; *** Professora Adjunta, Disciplina de Bioestatística, EPM.

Dr. Jaão Antonio Maciel Nóbrega - Disciplina de Neurologia, Escola Paulista de Medicina Rua Botucatu 740 - 04023 São Paulo SP - Brasil. 


\section{MATERIAL E METODOS}

Foram estudadas 101 pessoas, 53 do sexo masculino $(52,5 \%)$ e 48 do sexo feminino (47,5\%). A idade variou de 16 a 81 anos, sendo a média de 44 anos. Todas as pessoas foram previamente submetidas a exame clínico e foram avaliadas somente aquelas saudáveis, nas quais foram definitivamente afastadas quaisquer patologias que pudessem primária ou secundariamente comprometer o sistema nervoso periférico. Foi utilizado eletromiógrafo TECA TD-10. A temperatura ambiente foi controlada $\left(20-23{ }^{\circ} \mathrm{C}\right)$, assim como a temperatura cutanea $\left(30-32{ }^{\circ} \mathrm{C}\right)$ em membros superiores o (29-30 $9 \mathrm{C})$ em membros inferiores.

Condução sensitiva - Foi utilizada a técnica ortodrômica e avaliada a amplitude do potencial registrado, a latência e a velocidade de condução. Nos membros superiores foram estudados os nervos medianos, ulnares e radiais e, nos membros inferiores, os nervos surais e tibiais posteriores. A faixa de filtragem fol de 20 Hertz e $2 \mathrm{kHertz}$ e a varredura foi mantida em $2 \mathrm{~ms}$ por divisão. A intensidade de estímulo foi aumentada gradativamente até que se obtivesse a amplitude máxima do potencial registrado. A latência foi medida do início do artefato de estímulo até o início da deflexão negativa do potencial evocado. $O$ eletrodo terra foi fixado entre o eletrodo de estímulo e o de captação. Para o nervo mediano o eletrodo estimulador utilizado foi de anel, sendo o catodo colocado no terço proximal da primeira falange e o anodo, $2 \mathrm{~cm}$ distalmente. As medidas df; condução foram analisadas estimulando o 29, 39 e 4p dedos. Para o registro do potencial foram utilizados eletrodos de superficie (dois discos montados em barra fixa separados $2 \mathrm{~cm}$ entre si) situados sobre o trajeto do nervo mediano, logo acima da prega do punho, entre os tendóes dos músculos palmar longo e flexor radial do carpo. Foi também analisada a velocidade de condução sensitiva do nervo mediano, estimulando_o na palma da mão e registrando o potencial a nível do punho (condução palma-punho). Para o estudo da condução sensitiva no nervo ulnar foi utilizada técnica semelhante, porém os eletrodos de estimulação foram colocados no $5^{\circ}$ dedo e os de registro sobre o nervo ulnar na região medial do punho. O nervo radial foi estimulado na tabaqueira anatômica, sobre o tendão do músculo extensor longo do polegar e o potencial registrado com os eletrodos de captação localizados na transição entre do terço médio e distal da face lateral do antebraço. O nervo sural foi estimulado na regiâo retromaleolar exteını e o eletrodo de registro localizado no terço distal lateral da perna. $O$ nervo tibial posterior foi estimulado com eletrodos de anel colocados no hálux com o catodo situado na falange proximal e o arodo $2 \mathrm{~cm}$ distalmente, os eletrodos de registro sendo fixados na região retromaleolar interna.

condução motors - No estudo da condução motora foram avaliadas as amplitudes, as latências distais dos potenciais musculares compostos registrados e as medidis das velocidades de conduçāo. Nos membros superiores foram avaliados os nervos medianos, ulnares e radiais e, nos membros inferiares, as nervos fibulares e tibiais posteriores. A faixa de filtragem fo mantida entre 20 Hertz e $2 \mathrm{kHertz}$, varredura de tela em $5 \mathrm{~ms}$ por divisăo. A duraçă do estímulo variou de $0,05 \mathrm{~ms}$ a $0,2 \mathrm{~ms}$, conforme a necessidade para a obtençăo de resposta supramáxima. O eletrodo de estimulação utilizado é composto de duas barras fixas em forma de garra, separadas por $2 \mathrm{~cm}$ entre si. Os eletrodos de captação são constituídos de um par de discos de metal. o eletrodo terra sempre foi mantido entre os pontos de estímulo e eletrodo de captação. Para o estudo do nervo mediano, o eletrodo ativo foi colocado na regiāo tenar e o eletrodo de referência, fixado junto à articulação metacarpofalangeana do polegar. Estímulos foram aplicados junto à prega do punho, no cotovelo, na axila e no ponto de Erb. O nervo ulnar foi estimulado no punho, abaixo e acima do cotovelo, na axila e no ponto de Erb, sendo os eletrodos de registro fixados na região hipotenar to ativo sobre o músculo abdutor do $5^{\circ}$ dedo e o referência na articulação metacarpofalangeana do $5^{\circ}$ dedo). $O$ cotovelo foi mantido com angulação de aproximadamente $75^{\circ}$. O nervo radial foi estimulado em três pontos (braço, axila e ponto de Erb) e os potenciais registrados no músculo extensor comum dos dedos. O nervo fibular foi estimulado no tornozelo medialmente ao tendão do músculo extensor longo dos dedas do pé e abaixo e acima da cabeça da fíbula. O registro foi feito no músculo extensor curto dos dedos do pé sendo o eletrodo de referência colocado sobre a articulação metatarsofalangeana do hálux. o nervo tibial posterior foi estimulado na regiăo retromaleolar e na fossa poplítea, com o registro sendo feito na regižo plantar com o eletrodo ativo no músculo abdutor do $5^{\circ}$ dedo e o indiferente na articulação metatarsofalangeana do $5^{\circ}$ dedo.

Métodos estatísticos - Para a análise dos resultados foram utilizados os seguintes testes estatísticos: (1) teste «t» de Student para duas amostras não independentes com o objetivo de comparar os lados direito e esquerdo, em relação aos valores da amplitude, latência distal e velocidade de condução sensitiva e motora; (2) análise de variancia a um critério para comparar os grupos etários considerados segundo os valores das variáveis referidas; quando mostrou diferença significante esta análise foi complementada pelo teste de contrastes de Scheffé. Em todos os testes fixou-se em 0,05 ou $5 \%(<0,05)$ nivel para rejeição da hipótese de nulidades. 


\section{RESULTADOS}

Nas tabelas 1 a 5 gão mostrados os resultados obtidos. Para a conduça sensitiva as amplitudes estão em uV, as velocidades em $\mathrm{m} / \mathrm{s}$ e as latências em ms. Para a condução motora as amplitudes estão em $\mathrm{mV}$, as velocidades em $\mathrm{m} / \mathrm{s}$ e as latências em $\mathrm{ms}$.

Tabela 1 - Amplitwdes sensitivas dos nervos medianos, ulnares, surais e tibiais pasteriores.

\begin{tabular}{|c|c|c|c|c|c|c|c|c|c|c|c|c|c|c|c|}
\hline \multirow{2}{*}{\multicolumn{2}{|c|}{ MPDIDA }} & \multicolumn{3}{|c|}{$<20$ anos } & \multicolumn{2}{|c|}{ 20-20 anos } & \multicolumn{2}{|c|}{$30-39$ anos } & \multicolumn{2}{|c|}{$40-49$ anos } & \multicolumn{2}{|c|}{ 50-59 anos } & \multicolumn{2}{|c|}{ 60-69 anos } & \multirow{2}{*}{$\frac{70 \text { anos }}{18.9 \pm 4 .}$} \\
\hline & & D & 30.2 & \pm 12.0 & 31.8 & \pm 9.5 & 33.1 & \pm 10.8 & 43.2 & \pm 8.3 & 38.0 & \pm 9.2 & 26.6 & \pm 6.0 & \\
\hline \multirow{7}{*}{$\begin{array}{l}\text { 量 } \\
\text { 悬 } \\
\text { 息 }\end{array}$} & II DODO & $\mathbf{E}$ & 33.7 & \pm 13.3 & 35.1 & \pm 12.6 & 34.4 & \pm 10.8 & 43.4 & \pm 8.8 & 38.4 & \pm 8.8 & 27.5 & \pm 6.0 & $18.7 \pm 25$ \\
\hline & MLITUEE & D & 33.3 & \pm 12.8 & 32.2 & \pm 8.7 & 31.9 & \pm 12.0 & 42.8 & \pm 8.8 & 39.2 & \pm 8.8 & 25.7 & \pm 4.8 & $18.4 \pm 42$ \\
\hline & III D500 & $E$ & 33.7 & \pm 11.7 & 37.4 & \pm 16.3 & 34.1 & \pm 11.5 & 42.8 & \pm 8.9 & 38.9 & \pm 9.1 & 26.6 & \pm 5.0 & $17.4 \pm 2.7$ \\
\hline & APLITUDE & D & 32.7 & \pm 13.0 & 28.8 & \pm 9.6 & 28.3 & \pm 11.3 & 36.6 & \pm 8.2 & 35.5 & \pm 8.3 & 24.2 & \pm 4.8 & $16.8 \pm 3 A$ \\
\hline & IV DEDO & $\mathbf{E}$ & 34.3 & \pm 14.7 & 29.8 & \pm 10.1 & 28.8 & \pm 11.0 & 36.7 & \pm 9.0 & 34.5 & \pm 9.5 & 25.3 & \pm 4.6 & $15.6 \pm 2 A$ \\
\hline & AMPLITIOE & D & 131.7 & \pm 44.0 & 149.0 & \pm 53.1 & 152.0 & \pm 78.2 & 160.2 & \pm 55.7 & 124.0 & \pm 36.9 & 107.3 & \pm 44.0 & $71.8 \pm 30 \mathrm{~A}$ \\
\hline & PRIMA-PUKAO & $\mathbf{E}$ & 131.7 & \pm 40.2 & 161.5 & \pm 89.0 & 155.8 & \pm 84.8 & 158.3 & \pm 53.2 & 127.6 & \pm 28.7 & 107.3 & \pm 33.3 & $71.2 \pm 27.6$ \\
\hline \multirow{4}{*}{ 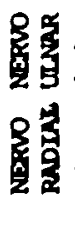 } & ANPLITUDE & D & 18.3 & \pm 4.7 & 20.2 & \pm 8.2 & 18.9 & \pm 3.9 & 27.7 & \pm 11.5 & 17.2 & \pm 4.7 & 16.5 & \pm 2.5 & $11.8 \pm 2 A$ \\
\hline & DEDOO & $\mathbf{E}$ & 20.7 & \pm 5.2 & 20.3 & \pm 3.8 & 18.8 & \pm 3.2 & 28.1 & \pm 10.7 & 17.7 & \pm 5.0 & 17.8 & \pm 3.5 & $12.7 \pm 22$ \\
\hline & & D & 42.5 & \pm 6.1 & 40.0 & \pm 14.2 & 52.2 & \pm 27.6 & 45.0 & \pm 9.4 & 54.7 & \pm 20.5 & 26.8 & \pm 5.3 & $25.8 \pm 49$ \\
\hline & & $\mathbf{E}$ & 43.3 & \pm 6.0 & 42.1 & \pm 16.2 & 49.1 & \pm 24.8 & 47.9 & \pm 10.5 & 53.5 & \pm 21.2 & 26.7 & \pm 5.2 & $26.5 \pm 43$ \\
\hline \multirow{4}{*}{ 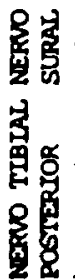 } & MPLITDE & D & 20.5 & \pm 8.2 & 24.7 & \pm 9.4 & 23.1 & \pm 12.4 & 25.8 & \pm 11.9 & 19.4 & \pm 8.7 & 24.1 & \pm 5.8 & $19.8 \pm 7.3$ \\
\hline & & 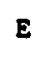 & 18.8 & \pm 7.3 & 23.7 & \pm 10.0 & 22.6 & \pm 12.8 & 27.5 & \pm 12.4 & 20.3 & \pm 9.3 & 23.9 & \pm 4.3 & $19.6 \pm 7.3$ \\
\hline & MPLTTUEE & D & 5.8 & \pm 1.6 & 6.4 & \pm 2.5 & 7.2 & \pm 3.8 & 6.8 & \pm 2.2 & 5.1 & \pm 1.5 & 7.7 & \pm 0.7 & - \\
\hline & & $\varepsilon$ & 7.0 & \pm 2.0 & 6.6 & \pm 2.2 & 7.0 & \pm 3.7 & $7-1$ & \pm 2.2 & 6.2 & \pm 2.0 & 1.0 & \pm 1.3 & - \\
\hline
\end{tabular}

Tabela 2 - Conduç̄o sensitiva dos nervos medianos, ulnares, nadiais, surais e tibiais posteriores.

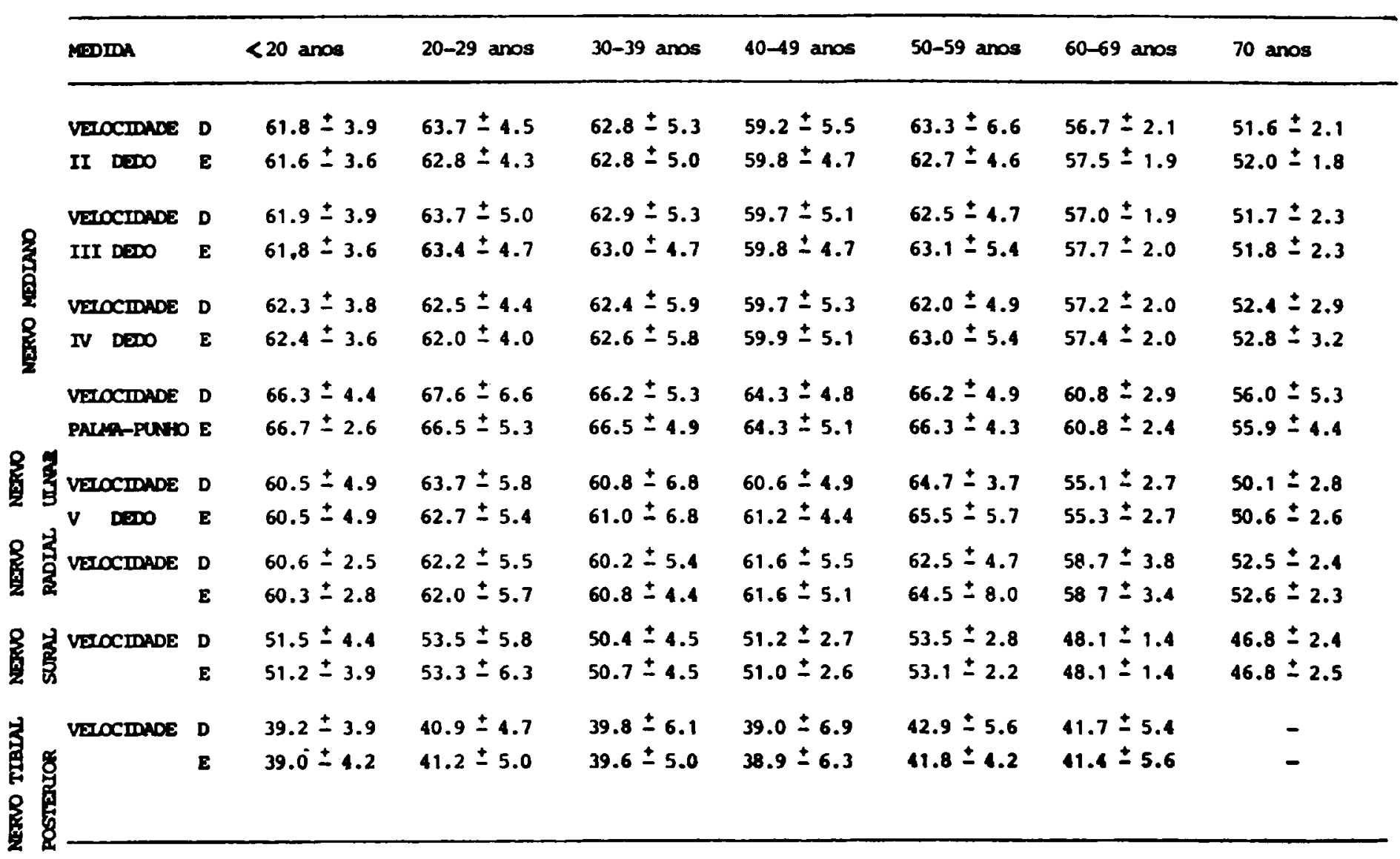


Tabela 3 - Conduço motora dos nervos mediano e radial.

\begin{tabular}{|c|c|c|c|c|c|c|c|c|c|}
\hline & MEDIDA & & $<20$ anos & $20-29$ anos & $30-39$ anos & $40-49$ anos & $50-59$ anos & $60-69$ anos & 70 anos \\
\hline \multirow{9}{*}{$\begin{array}{l}\text { 是 } \\
\text { 蚎 } \\
\text { 量 }\end{array}$} & AMPLITLDE & $\begin{array}{l}\text { D } \\
\text { E }\end{array}$ & $\begin{array}{l}22.5 \pm 4.8 \\
25.0 \pm 4.4\end{array}$ & $\begin{array}{l}23.5 \pm 6.2 \\
23.5 \pm 5.9\end{array}$ & $\begin{array}{l}18.7 \pm 3.9 \\
18.5 \pm 3.8\end{array}$ & $\begin{array}{l}21.4 \pm 4.6 \\
21.3 \pm 4.3\end{array}$ & $\begin{array}{l}18.4 \pm 3.2 \\
17.5 \pm 3.3\end{array}$ & $\begin{array}{l}13.4 \pm 2.9 \\
13.4 \pm 2.8\end{array}$ & $\begin{array}{l}10.2 \div 2.1 \\
10.2 \pm 2.1\end{array}$ \\
\hline & LATENCIA & D & $3.7 \pm 0.3$ & $3.3 \pm 0.5$ & $3.2 \pm 0.4$ & $3.0 \pm 0.3$ & $2.9 \pm 0.3$ & $3.3 \pm 0.5$ & $3.9 \pm 0.2$ \\
\hline & DISTAL & E & $3.7 \pm 0.3$ & $3.2 \pm 0.5$ & $3.2 \pm 0.3$ & $3.0 \pm 0.3$ & $2.7 \pm 0.4$ & $3.2 \pm 0.5$ & $3.9 \pm 0.2$ \\
\hline & VEIOCIDADE & D & $58.3 \pm 4.3$ & $58.3 \pm 4.4$ & $58.4 \pm 4.1$ & $58.1 \pm 2.5$ & $59.6 \pm 1.4$ & $56.3 \pm 2.0$ & $57.6 \div 3.0$ \\
\hline & ANTEBRAQD & $E$ & $57.9 \pm 4.4$ & $58.2 \pm 4.8$ & $57.7 \pm 4.0$ & $57.9 \pm 2.3$ & $59.8 \pm 2.3$ & $56.2-2.2$ & $52.3 \div 2.6$ \\
\hline & VEIOCIDADE & D & $62.7 \pm 4.1$ & $65.2 \pm 3.9$ & $64.0 \pm 6.0$ & $65.3 \pm 5.3$ & $65.5 \pm 5.8$ & $61.4 \pm 3.2$. & $57.5 \pm 4.3$ \\
\hline & BRAQD & E & $63.5 \pm 4.1$ & $65.8 \pm 4.0$ & $64.5 \pm 5.6$ & $65.8 \pm 4.4$ & $65.9 \pm 6.8$ & $61.8 \pm 2.5$ & $57.9 \pm 4.4$ \\
\hline & VETOCIDADE & D & $73.3 \pm 3.4$ & $77.8 \pm 6.6$ & $74.4 \pm 6.0$ & $73.7 \pm 4.8$ & $73.8 \pm 6.1$ & $68.5 \pm 3.2$ & $64.5 \pm 4.3$ \\
\hline & AXIIA & $\mathbf{E}$ & $77.1 \pm 4.9$ & $77.2 \pm 5.2$ & $74.7 \pm 6.5$ & $73.2 \pm 3.2$ & $72.9 \pm 4.7$ & $68.5 \pm 2.4$ & $64.5 \pm 3.1$ \\
\hline \multirow{6}{*}{ 量 } & AMPLTIDE & D & $19.5 \pm 4.4$ & $15.3 \pm 5.5$ & $14.3 \pm 3.7$ & $18.0 \pm 2.8$ & $15.5 \pm 2.5$ & $13.4 \pm 2.5$ & $11.5 \pm 2.3$ \\
\hline & & $\mathbf{E}$ & $19.3 \pm 4.5$ & $15.6 \pm 5.3$ & $14.5 \pm 3.6$ & $18.1 \pm 2.9$ & $15.5 \pm 2.5$ & $13.3 \pm 2.5$ & $11.6 \pm 2.2$ \\
\hline & VEIOCIDADE & D & $67.0 \pm 2.7$ & $69.5 \pm 6.7$ & $66.9 \pm 8.5$ & $64.1 \pm 7.1$ & $65.1 \pm 8.1$ & $58.3 \pm 3.5$ & $59.2 \pm 4.8$ \\
\hline & BRACO & $\mathbf{E}$ & $65.3 \pm 3.4$ & $69.7 \pm 7.0$ & $65.9 \pm 7.7$ & $63.5 \pm 6.2$ & $64.3 \pm 5.7$ & $58.8 \pm 4.3$ & $58.9 \pm 4.3$ \\
\hline & VEIOCIDADE & D & $79.1 \pm 6.0$ & $80.5 \pm 6.0$ & $82.5 \pm 6.4$ & $77.4 \pm 6.3$ & $73.9 \pm 7.6$ & $69.2 \pm 3.7$ & $67.0 \pm 3.8$ \\
\hline & AXIIA & $\mathbf{E}$ & $78.9 \pm 5.6$ & $79.7 \pm 5.7$ & $81.5 \pm 6.0$ & $78.6 \pm 6.5$ & $73.4 \pm 7.8$ & $68.7 \pm 4.4$ & $67.2 \pm 4.4$ \\
\hline
\end{tabular}

Tabela 4 - Conducão motora do nerva ulnar.

\begin{tabular}{|c|c|c|c|c|c|c|c|c|c|c|}
\hline & MEDIDA & & $<20$ anos & 20-29 anos & 30-39 anos & $40-49$ aros & 50-59 anos & $60-69$ anos & 70 ans & \\
\hline \multirow{12}{*}{ 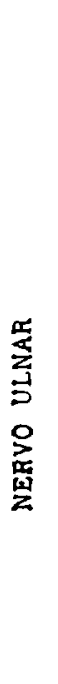 } & \multirow[t]{2}{*}{ AMPLTTUDE } & D & $17.8 \pm 2.8$ & $18.3 \pm 4.7$ & $16.6 \pm 2.5$ & $17.8 \pm 2.8$ & $14.7 \pm 2.8$ & $11.0 \pm 3.1$ & 8.5 & \pm 2.9 \\
\hline & & $\mathbf{E}$ & $17.6 \pm 3.2$ & $18.3 \pm 4.2$ & $16.3 \pm 2.5$ & $17.6 \pm 3.2$ & $14.9 \pm 2.9$ & $11.0 \pm 3.1$ & 8.6 & \pm 2.9 \\
\hline & IATENCIA & D & $2.8 \pm 0.4$ & $2.6 \pm 0.5$ & $2.5 \pm 0.3$ & $2.4 \pm 0.4$ & $2.3 \pm 0.2$ & $2.5 \pm 0.3$ & 2.7 & \pm 0.5 \\
\hline & DISTAL & $\mathbf{E}$ & $2.7 \pm 0.3$ & $2.7 \pm 0.5$ & $2.5 \pm 0.4$ & $2.4 \pm 0.3$ & $2.3 \pm 0.1$ & $2.5 \pm 0.3$ & 2.6 & \pm 0.4 \\
\hline & VETOCmADE & D & $54.3 \pm 5.8$ & $58.3 \pm 5.2$ & $58.4 \pm 4.3$ & $56.8 \pm 3.2$ & $55.0 \pm 4.5$ & $55.0 \pm 4.5$ & 53.5 & $\pm 3,0$ \\
\hline & AMTIEBRAgO & $\boldsymbol{E}$ & $54.7 \pm 5.6$ & $58.1 \pm 5.1$ & $58.6 \pm 4.2$ & $57.0 \pm 3.2$ & $56.3 \pm 2.8$ & $55.3 \pm 4.4$ & 52.5 & \pm 2.6 \\
\hline & VETOCIDADE & D & $61.7 \pm 5.7$ & $64.5 \pm 5.3$ & $62.8 \pm 4.2$ & $62.5 \pm 4.1$ & $60.2 \pm 3.8$ & $59.0 \pm 4.6$ & 58.0 & \pm 5.0 \\
\hline & COTOVELO & $E$ & $61.6 \pm 5.8$ & $63.5 \pm 4.8$ & $63.1 \pm 3.8$ & $62.2 \pm 4.0$ & $61.0 \pm 3.4$ & $58.3 \pm 4.6$ & 57.1 & \pm 3.1 \\
\hline & VEIOCIDADE & D & $66.7 \pm 5.3$ & $69.1 \pm 4.8$ & $67.5 \pm 3.5$ & $66.5 \pm 5.0$ & $65.6 \pm 3.5$ & $66.3 \pm 4.2$ & 62.3 & \pm 4.5 \\
\hline & BRAQD & $\mathbf{E}$ & $66.6 \pm 4.9$ & $67.8 \pm 4.7$ & $68.0 \pm 3.2$ & $66.5 \pm 4.5$ & $66.5 \pm 3.2$ & $65.6 \pm 4.2$ & 60.1 & \pm 3.7 \\
\hline & VEIOCIDADE & D & $72.3 \pm 2.2$ & $76.0 \pm 4.2$ & $74.6 \pm 4.5$ & $73.1 \pm 4.4$ & $74.2 \pm 5.2$ & $69.7 \pm 3.8$ & 66.5 & \pm 5.0 \\
\hline & AXIIA & $\mathbf{E}$ & $71.2 \pm 1.9$ & $76.2 \pm 4.9$ & $74.9 \pm 4.2$ & $73.7 \pm 3.3$ & $73.0 \pm 5.0$ & $69.5 \pm 3.3$ & 65.9 & \pm 4.8 \\
\hline
\end{tabular}


Tabela 5 - Conducāo motora dos nervos fibular e tibial posterior.

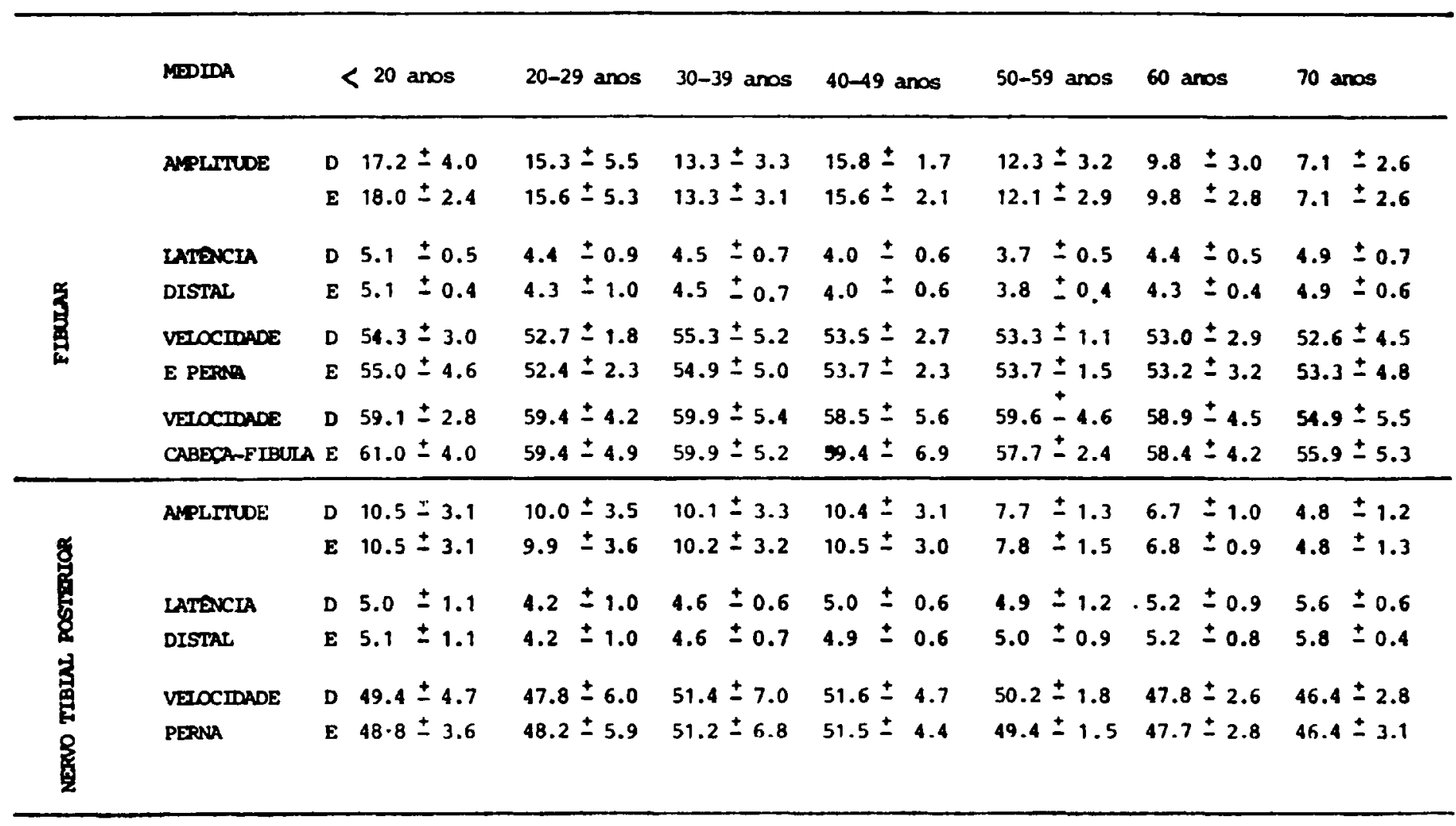

\section{COMERTARIOS}

Em nosso estudo não foram evidenciadas diferenças significativas das amplitudes dos potenciais sensitivos entre os lados direito e esquerdo. Em relação à idade, verificou-se que houve decréscimo nos grupos de maior faixa etária, para os nervos sural e tibial posterior não havendo variação significativa. Os valores das amplitudes dos potenciais sensitivos encontrados foram maiores que os referidos por Dawson 6 , Cruz Martinez e col.4 e por Vandendriesche e col.25.

Com relação às velocidades de condução sensitiva, também não houve diferença entre os dados obtidos para os lados direito e esquerdo e houve redução nas faixas etárias mais elevadas. Este fato também foi citado por Vandendriesche e col.25, Feibel e Foca 8 , Cruz Martinez e col.4 e Miglietta 19. Outros autores, como Truong e col.24 e Behse e Buchtal 1 chegaram às mesmas conclusões, fazendo análises em diferentes nervos e com diferentes parámetros de estudo.

Em relação à amplitude do potencial muscular, não houve diferenças significativas entre os vários grupos examinados. Já a laténcia motora terminal foi maior no grupo de pessoas mais idosas, nos nervos mediano e tibial posterior bilateralmente, sem diferenças significativas quanto à lateralidade. A velocidade de condução motora foi menor nos segmentos distais que nos proximais, tanto nos nervos examinados nos membros superiores como nos dos membros inferiores. Este fato também é referido na literatura. Os valores obtidos nos nervos dos membros inferiores foram menores que aqueles obtidos nos membros superiores, constatação também referida por Buchtal e Rosenfalck 2 e por Gassel e Diamantopoulos 9 .

Na literatura já havia citação de estudos com diferentes grupos raciais, como os realizados por Thies e col.23 e Sing e Maini 22 em pessoas de origem indiana e por Longe 18, que estudou um grupo de nigerianos. Esses autores năo encontraram diferenças significativas entre seus resultados e os obtidos por outros autores.

Os valores obtidos em nosso estudo não podem ser camparados estatisticamente aos demais valores referidos na literatura porque este foi o único em que se estudaram vários nervos na mesma pessoa e em ambos os lados Além disso, as faixas etárias foram bastante restritas e diferentes das estudadas nas pesquisas encontradas na literatura. Apesar desse fato, os dados obtidos em nosso material são concordantes aos obtidos por outros autores, mostrando: redução da velocidade de condução 
nervosa nos segmentos distais em relação aos proximais, tanto em nervos de membros superiores como em nervos de membros inferiores; valores maiores da velocidade de condução nervosa nos nervos examinados nos membros superiores que nos dos membros inferiores; redução da velocidade de condução nervosa e amplitude com o passar da idade.

\section{REFERENCIAS}

1. Behse F, Buchthal F. Normal sensory conduction in the nerves of the leg in men J Neurol Neurosurg Psychiatry 1971, 34:404-414.

2. Buchthal F, Rosenfalck A. Evoked action potentials and conduction velocity in human sensory nerves. Brain Res 1966, 3:1-119.

3. Checkles NS, Russakov AD, Piero DL. Ulnar nerve conduction velocity: effect of elbow position on measurement. Arch Phys Med Rehabil 1971, 52:362-365.

4. Cruz Martinez A, Guitierrez AM, Perez MC. Estudio de la velocidad de conduccion del nervio sural in individuos normales de diferentes. edades Rev Clin Esp 1977, 144:277-282.

5. De Jong RH, Hershey WN, Wiagman IH. Nerve conduction velocity during hypotermia in man. Anesthesiology 1966, 27:805-810.

6. Dawson GD. The relative excitability and conduction velocity of sensory and motor nerve fibers in man. $J$ Physiol 1956, 131:436-451.

7. Dawson GD, Scott JW. The recording of nerve action potentials through the skin in man. J Neurol Neurosurg, Psychiatry 1949, 12:259-267.

8. Feibel A, Foca FJ. Sensory conduction of radial nerve. Arch Phys Med Rehabil 1974, 55 :314_316.

9. Gassel MM, Diamantopoulos E. Pattern of conduction times in the distribution of the radial nerve. Neurology 1964, 14:222-231.

10. Gilliatt RW, Thomas PK. Changes in nerve conduction with ulnar lesions at the elbow. J Neurol Neurosurg: Psychiatro 1960, 23:312.

11. Gilliatt $\mathrm{RW}$, Thomas PK. The recording of lateral popliteal nerve action potentials in man. J Neurol Neurosurg Psychiatry 1961, 24:305.

12. Halar EM, Delisa JA, Brozovich FV. Nerve conduction velocity: relationships of skin, subcutaneous and intramuscular temperatures. Arch Phys Med Rehabil 1980, 61:199-203.

13. Halar EM, Delisa JA, Brosovich FV. Peroneal nerve conduction velocity: the importance on temperature correction. Arch Phys Med Rehabil 1981, 62:439-443.

14. Halar EM, Delisa JA, Soine TL. Nerve conduction studies in upper extremities: skin temperature corrections. Arch Phys Med Rehabil 1983, 64:412-416.

15. Harding C, Halar E. Motor and sensory ulnar nerve conduction velocities: effect of elbow position. Arch Phys Med Rehabil 1983, 64:227-232.

16. Kimura J. Electrodiagnosis in Diseases of Nerve and Muscle: Principles and Practice. Philadelphia: FA Davis, 1983.

17. La Fratta CW, Canestrari RE. A comparision of sensory and motor nerve conduction velocities as related to age. Arch Phys Med Rehabil 1966, 47:286_290.

18. Longe AC. Sensory conduction velocities in healthy Nigerian adults. Afr J Med Sci 1983, $12: 173-176$.

19. Miglietta $O E$. Sensory conduction of digital nerve fibers. Am J Phys Med 1969, 48:78-84.

20. Nelson RM. Effects of elbow position on motor conduction velocity of the ulnar nerve. Phys Ther 1980, 60:780-783.

21. Simpson JA. Electrical signs in the diagnosis of carpal tunnel and related syndromes. $\mathrm{J}$ Neurol Neurosurg Psychiatry 1956, 19:275.

22. Sing PI, Maini BK. Motor conduction velocities of peripheral nerves in normal subjects. Indian $J$ Med Sci 1970, 34:144-147.

23. Thies RH, Billinghurst JR, Richardson HD. Motor nerve conduction velocities in healthy young east Africans. J Appl Physiol 1967, 23:321-323 .

24. Truong XT, Russo FI, Vagi I, Rippel DV. Conduction velocity in the proximal sural nerve. Arch Phys Med Rehabil 1979, 60:304-308.

25. Vlandendriesche $G$, Vanhecke $J$, Rosselle $N$. Normal sensory conduction in the distal segment of the median and radial nerve: relation to age. Electromyogr Clin Neurophysiol 1981, 21:511-519. 